\title{
Dielectric Constant Measurement on Calcium and Lanthanum Doped Triglycine Sulphate Crystals
}

\author{
P. Manoharan ${ }^{1 *}$ and N. Neelakanda Pillai ${ }^{2}$ \\ ${ }^{I}$ Department of Physics, Udaya Polytechnic College, Vellamodi, Tamilnadu, India \\ ${ }^{2}$ Department of Physics, Arignar Anna College, Aralvoimozhi, Tamilnadu, India
}

\begin{abstract}
Triglycine Sulphate (TGS) salts were synthesized. Calcium and lanthanum doped TGS crystals were grown from aqueous solutions by slow evaporation technique. The dielectric constant and AC conductivity measurement were carried out at various temperature ranging from $30^{\circ} \mathrm{C}$ to $120^{\circ} \mathrm{C}$ at different frequencies, the variation of dielectric constant with temperature and frequency were studied and it is found that the dielectric constant values decreases with increase in frequency and the AC conductivity increases with increase in frequency.
\end{abstract}

Keyword: TGS, Dielectric constant, rare earth, AC conductivity

\section{Introduction}

Triglycine sulphate (TGS) crystal is a useful ferroelectric material founded in 1956 and has low dielectric constant and large pyroelectric coefficient.It finds applications in the fabrication of pyroelectric vidicon tubes, capacitors, transducers, sensors and also it is used in the infra red detection techniques as commercial product [1-2]. TGS crystal shows a ferroelectric phase transition at the curie point temperature $(\mathrm{Tc}=$ $49^{\circ} \mathrm{C}$ ). Below the Tc, TGS possesses the polar point symmetry of group 2 of monoclinic system, spontaneous polarization arises along the $b$-axis and above Tc, it possesses the non-polar point group $2 / \mathrm{m}$ of the monoclinic system[3-4].

Stankowska et al [5] have studied the dielectric properties of TGS crystals admixture with D and DLphenylalanine. Michnevick and Kashevich [6] have studied the effect of impurity concentration gradients on the dielectric properties of triglycine sulphate crystals with the non isomorphic impurity ions of chromium and mixed isomorphic crystals of TGS and TG selenate. R.Muralidharan et al [7] have studied the temperature dependence of dielectric constant for Pure and rare earth doped TGS crystals and they have found that, the ferroelectric phase transition occurs at $49^{\circ} \mathrm{C}$ and there was no change in the transition temperature due to dopant addition. K.Balasubramanian et al [8] have also found that the ferroelectric phase transition occurs at $49^{\circ} \mathrm{C}$ for pure and copper sulphate doped TGS at $1 \mathrm{KHz}$ Frequency.

\section{Experimental Details}

The pure and calcium doped TGS crystals were grown by slow evaporation technique as explained in the previous paper [9].

The capacitance and dielectric loss factor $(\tan \delta)$ measurement were carried out by "Agilant LCR meter" for the frequencies viz. $50 \mathrm{~Hz}, 100 \mathrm{~Hz}, 1 \mathrm{KHz}, 10 \mathrm{KHz}, 50 \mathrm{KHz}, 100 \mathrm{KHz}, 200 \mathrm{KHz}$ at various temperatures ranging from $30^{\circ} \mathrm{C}$ to $120^{\circ} \mathrm{C}$ while cooling the sample. Temperature was controlled to an accuracy available. Air capacitance $\left(\mathrm{C}_{\text {air }}\right)$ also measured for the thickness equal to that of the sample crystal. The sample cleaved perpendicular to the polar axis (b-axis), with dimension $\left(6 \times 6 \times 2 \mathrm{~mm}^{3}\right)$ were used. The sample crystals were polished and opposite faces were coated with good quality graphite to obtain a good conductive surface layer . They were annealed for two hours at approximately equal to $120^{\circ} \mathrm{C}$ to remove moisture content if present.

The dimension of the sample crystal were measured using a travelling microscope $(\mathrm{LC}=0.001 \mathrm{~cm})$ the dielectric constant of the crystal was calculated using the relation [10] (as the crystal area was smaller than the plate area of the cell)

$\varepsilon_{\mathrm{r}=}[$ Ccrys-Cair(1-Acrys/Aair) (Aair/Acrys)] / Cair

where, $\mathrm{C}_{\text {crys }}$ is the capacitance of the crystal (including air), $\mathrm{C}_{\text {air }}$ is the capacitance of the air, $\mathrm{A}_{\text {crys }}$ is the area of the crystal touching the electrode and $\mathrm{A}_{\text {air }}$ is the area of the electrodes.

The AC conductivity $\left(\sigma_{\mathrm{ac}}\right)$ was calculated using the relation [10]

$\sigma_{\mathrm{ac}}=\varepsilon_{\mathrm{r}} \varepsilon_{0} \omega \tan \delta$

Where $\varepsilon_{0}$ is the permittivity of free space $\left(8.85 \times 10^{-12} \mathrm{C}^{-2} \mathrm{~N}^{-1} \mathrm{~m}^{-2}\right)$ and $\omega$ is the angular frequency $(\omega=2 \Pi \mathrm{f})$, $\mathrm{f}$ is the frequency of $\mathrm{AC}$. The values were fitted in to the equation. 


\section{Results and Discussion}

Fig(i) shows the temperature dependence of dielectric constant at $1 \mathrm{KHz}$ frequency for the pure and calcium doped TGS crystals. Fig(ii) shows that the temperature dependence of dielectric constant at $1 \mathrm{KHz}$ frequency for the pure and Lanthanum doped TGS crystals. In both the cases the crystals have maximum dielectric constant value at curie point temperature $\left(\mathrm{T}_{\mathrm{c}}=50^{\circ} \mathrm{C}\right)$ in agreement with the reported values [7-8], the same result was obtained by [2] and [3] the doped TGS crystal have highest dielectric constant values than the pure TGS crystals. The peak value increases with increase in the dopant concentration and LaTGS crystals have highest dielectric constant value compared to the CaTGS ,The rapid increase in dielectric constant of the doped TGS crystals at the curie temperature is attributed to free charge carriers created by the dopant.

The variation of dielectric constant with frequency at $50^{\circ} \mathrm{C}$ for Calcium and Lanthanum doped TGS crystals are shown in fig(iii) and fig(iv) respectively. In both the cases the dielectric constant values decreases with increase in frequency . this may be due to the fact that at highest frequencies only electronic polarization will be present.

The variation of AC conductivity with temperature and frequency for CaTGS and LaTGS are shown in fig (v) to fig (viii). it is observed that the AC conductivity also has maximum value at Curie point temperature and increases with increase in frequency

\section{Conclusion}

The dielectric constant calculated for Pure and doped TGS crystals are low and has highest value at ferroelectric phase transition temperature. It varies inversely with frequency and AC conductivities also have maximum value at curie point temperature , and both vary with dopant addition

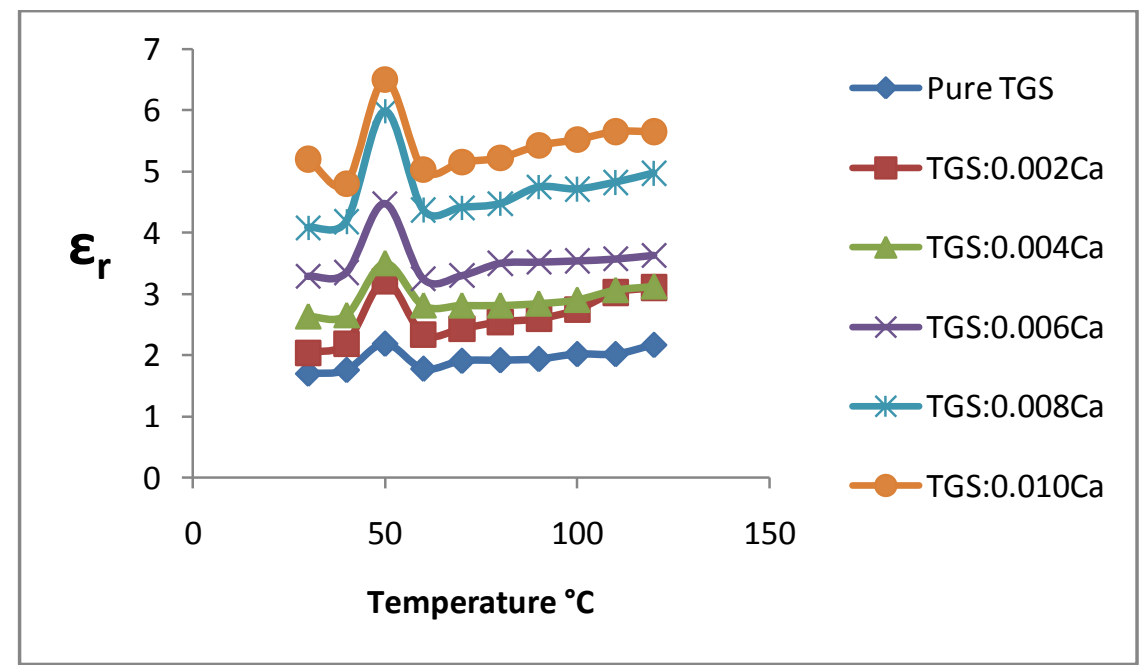

Fig (i ).Variation of Dielectric Constant with temperature at $1 \mathrm{KHz}$ Frequency for Pure and Ca doped TGS 


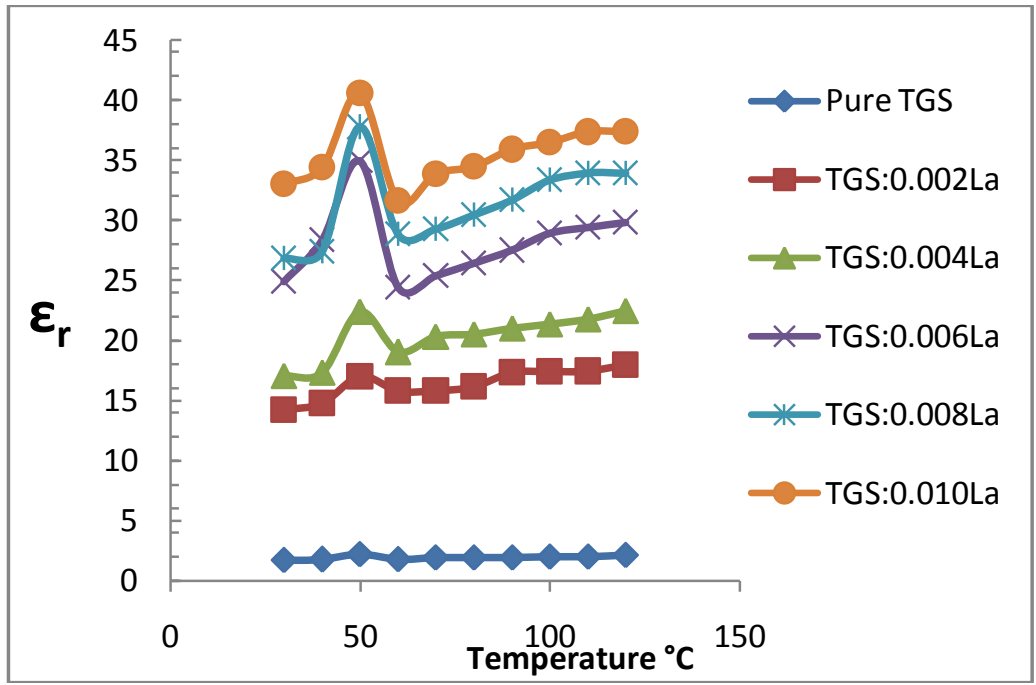

Fig (ii). Variation of Dielectric Constant with temperature at 1KHz Frequency for Pure and La doped TGS

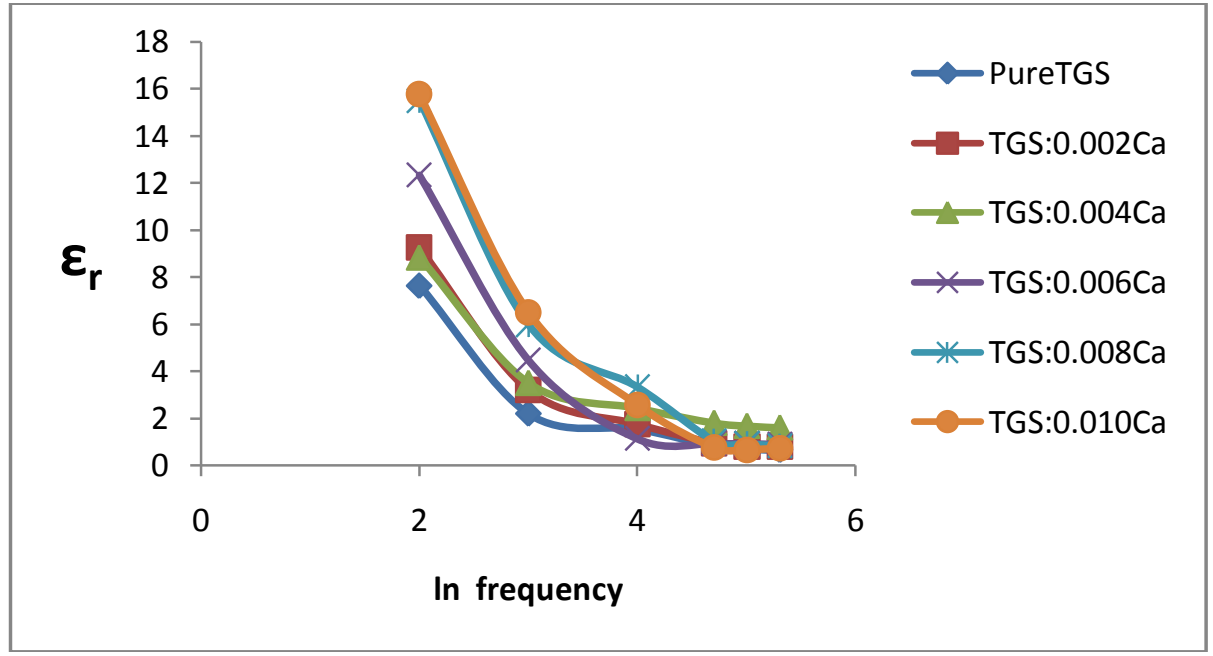

Fig(iii).Variation of dielectric Constant with frequency at $50^{\circ} \mathrm{C}$ for Pure and $\mathrm{Ca}$ doped TGS

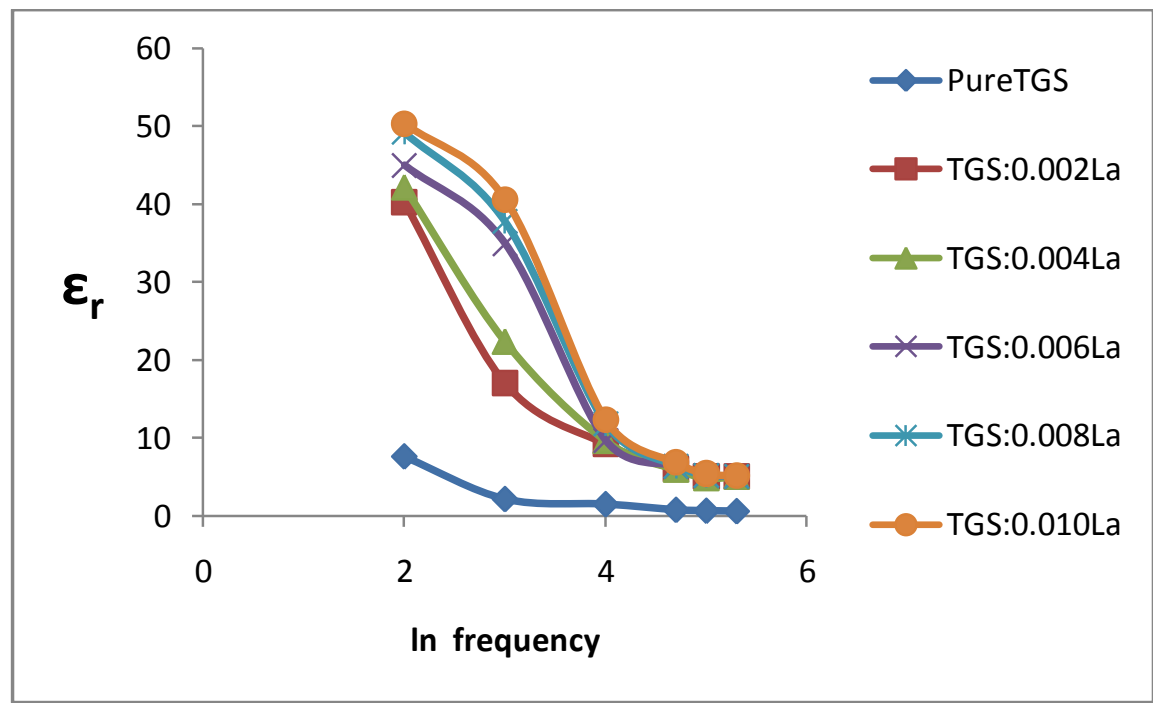

Fig(iv). Variation of dielectric Constant with frequency at $50^{\circ} \mathrm{C}$ for Pure and La doped TGS 


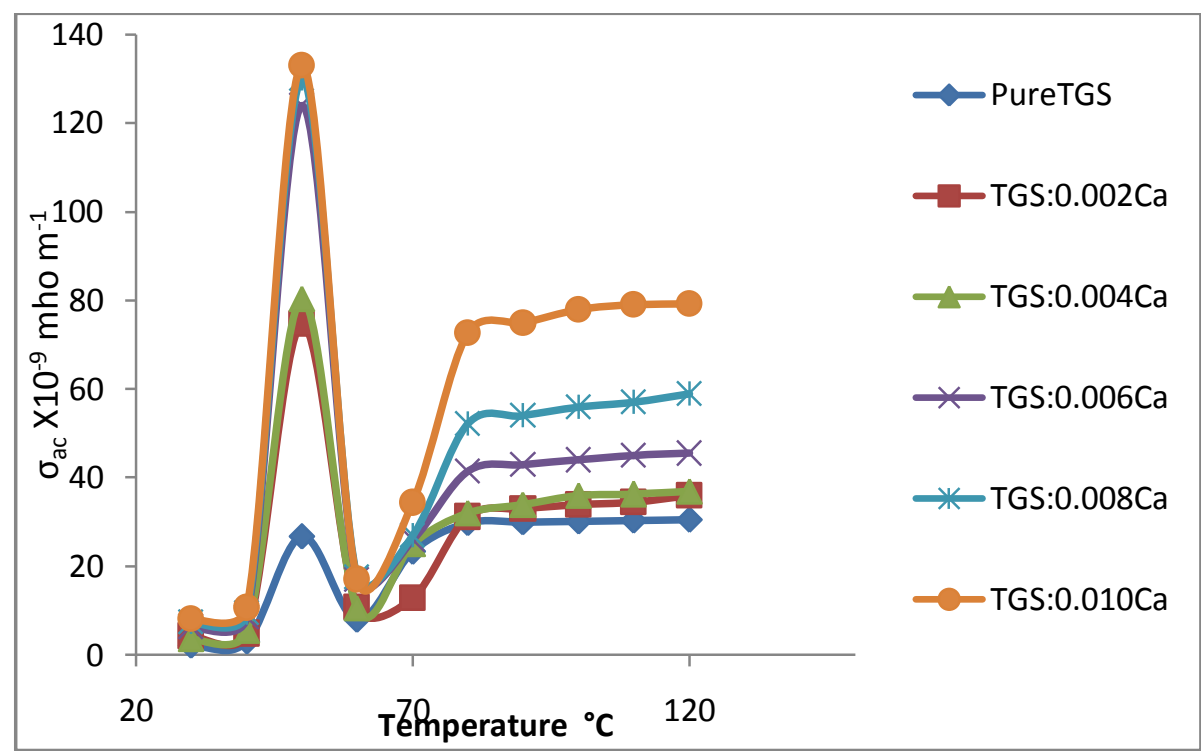

Fig (v). Variation of AC conductivity with Temperature at $1 \mathrm{KHz}$ frequency for Pure and Ca doped TGS

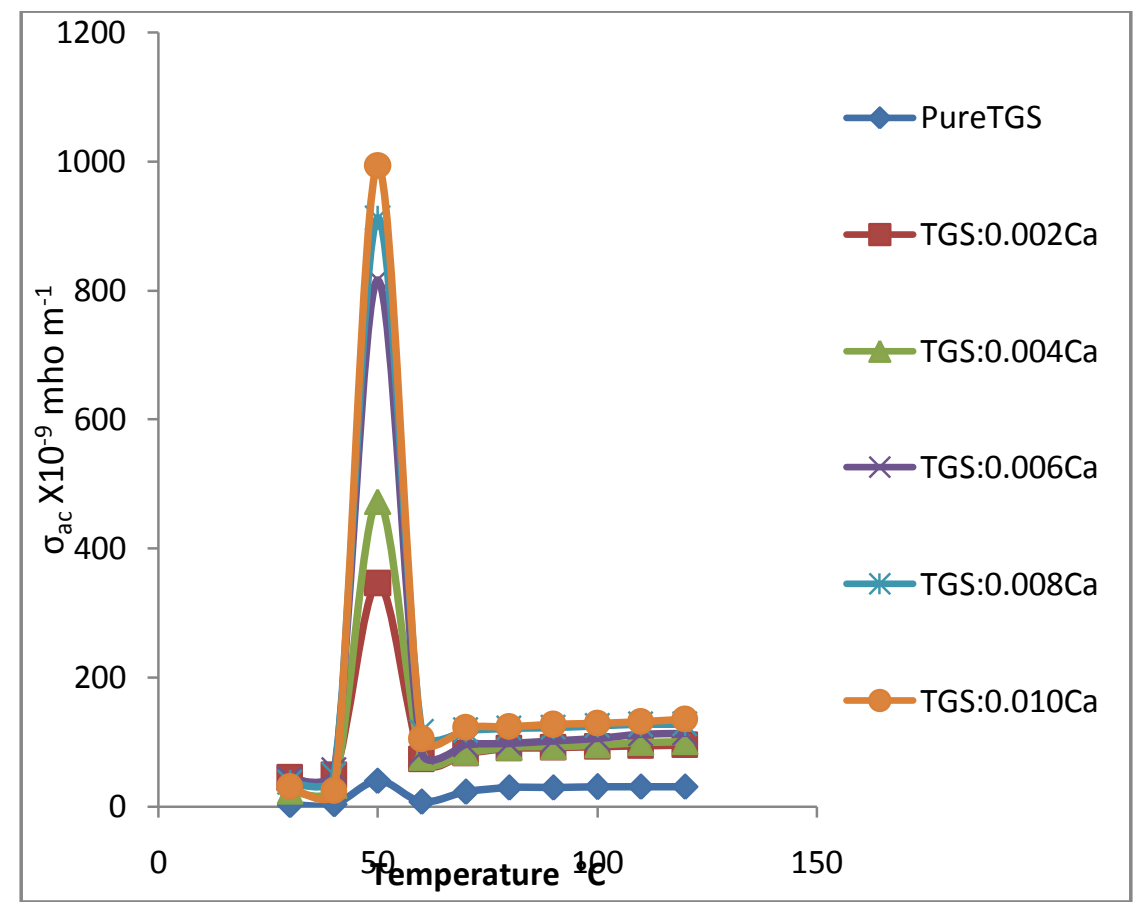

Fig(vi). Variation of AC conductivity with Temperature at $1 \mathrm{KHz}$ frequency for Pure and La doped TGS 


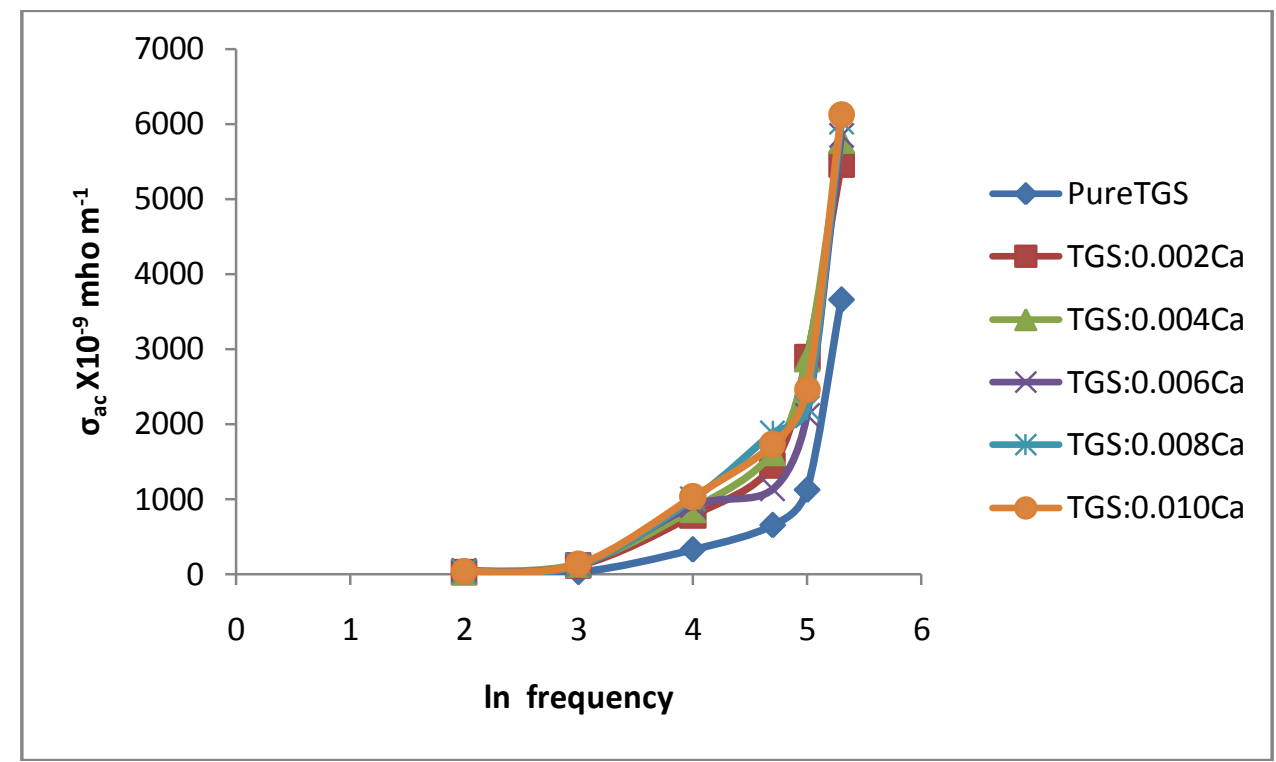

Fig(vii). Variation of AC Conductivity with frequency at $50^{\circ} \mathrm{C}$ for Pure and Ca doped TGS

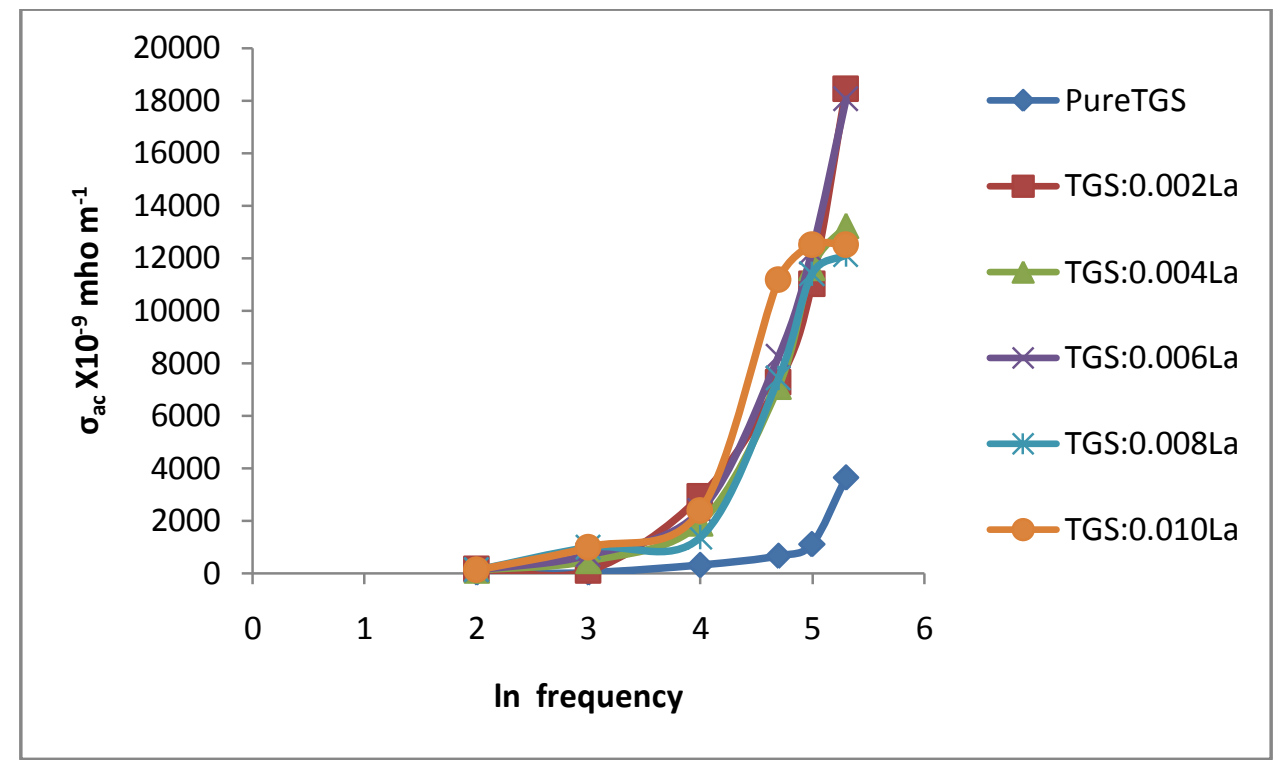

Fig(viii). Variation of AC Conductivity with frequency at $50^{\circ} \mathrm{C}$ for Pure and La doped TGS

\section{References}

[1] D.Sun, X.Yu, Q.Gu, cryst.,Res.Technol.34(1999),1255.

[2] T.Krajewski, T. Breczewki, Ferroelectrics 25 (1980) 547

[3] E.A. Wood, AN.Holden, Acta crystallogr.10(1957) 145

[4] H.Newman, H.Budzier, Ferroelectics 133(1992( 41)

[5] Stakowska.J., Peter.E., Trybula.M, Acta Physica polonica,97,(2000),1061-72.

[6] Mikhnevich.v.v, Kashevich.I.V., Sov.Tech. Physics, 18,(1992),43-5

[7] Muralidharan.R, Mohan Kumar.R, Ushasree.P.M, Jayavel.R, Ramasamy.P., J.cryst. Growth 234, (2002),545-50

[8] K.Balasubramanian, Recent research in scholar and Technology 2010,2(3);06-13

[9] P.Manoharan, N.Neelakanda pillai, Archives of Physics Research, 2013, 4 (2), 22-25

[10] N.Neelakanda Pillai,C.K.Mahadevan, Mater.Manuf.Processes 22 (2007) 393. 